\title{
A ENFERMAGEM CONSTRUINDO SIGNIFICADOS A PARTIR DE SUA INTERAÇÃO SOCIAL COM IDOSOS HOSPITALIZADOS ${ }^{1}$
}

\author{
Marinês Tambara Leite², Lucia Hisako Takase Gonçalves ${ }^{3}$
}

${ }^{1}$ Texto elaborado a partir da tese de doutorado defendida no Programa de Pós-Graduação em Gerontologia Biomédica da
Pontifícia Universidade Católica do Rio Grande do Sul (PUCRS), 2007.
${ }^{2}$ Doutora em Gerontologia Biomédica. Docente do Centro de Educação Superior do Norte do Rio Grande do Sul da Universidade
Federal de Santa Maria. Rio Grande do Sul, Brasil. E-mail: tambaraleite@yahoo.com.br
${ }^{3}$ Doutora em Enfermagem. Professor Associado do Departamento de Enfermagem e do Programa de Pós Graduação em
Enfermagem da Universidade Federal de Santa Catarina. Orientadora da tese. Santa Catarina, Brasil. E-mail: lucia@nfr.ufsc.br

RESUMO: A enfermagem é responsável pelo atendimento de todos os pacientes internados, inclusive os idosos, em hospital geral. $\mathrm{O}$ objetivo do estudo foi analisar o processo interacional da equipe de enfermagem que cuida de idosos internados em hospitais da $17^{\mathrm{a}}$ Coordenadoria Regional de Saúde/Secretaria Estadual de Saúde do Rio Grande do Sul. Pesquisa qualitativa interpretativa teve seus dados coletados por entrevista junto a 74 integrantes da equipe de enfermagem entre enfermeiros, técnicos e auxiliares de enfermagem de 14 hospitais. A análise se deu à luz do interacionismo simbólico resultando em categorias de significado das interações e de atitudes em: os profissionais da enfermagem, na vigência da falta de capacitação, processam o cuidado dos pacientes idosos hospitalizados a partir da forma como o fenômeno se apresenta em sua vida social; quanto à atitude, consideram a importância do preparo especializado em gerontogeriatria sem desconsiderar vocação pessoal para o cuidado de pessoas idosas como imprescindível.

DESCRITORES: Enfermagem geriátrica. Idoso. Cuidados de enfermagem. Hospitalização.

\section{NURSING CONSTRUCTING MEANINGS FROM ITS SOCIAL INTERACTION WITH THE HOSPITALIZED ELDERLY}

\begin{abstract}
Nursing is responsible for servicing all hospitalized patients, including the elderly, in general hospitals. The objective of this study was to analyze the interaction process from the nursing team which cares for elderly patients in hospitals of the 17th Health Regional Coordination/Health State Secretary/Rio Grande do Sul, Brazil. This interpretative and qualitative study collected its data through interviews with 74 nursing team members, including nurses, technicians, and nursing assistants in 14 hospitals. Data was analyzed in the light of symbolic interactionism and resulting in categories of meanings from interactions and attitude about: nursing professionals, lack of capacitance validity, process of caring hospitalized elderly patients from the way the phenomenon is in their social life; on the attitude, it is considered the importance of specialized training in gerontogeriatry without disregarding personal vocation to the care of the elderly as essential.
\end{abstract}

DESCRIPTORS: Geriatric nursing. Aged. Nursing care. Hospitalization.

\section{LA ENFERMERÍA EN EL DESARROLLO DE SIGNIFICADOS A PARTIR DE SU INTERACCIÓN SOCIAL CON LOS ANCIANOS HOSPITALIZADOS}

\begin{abstract}
RESUMEN: La enfermería es responsable por el cuidado de todos los pacientes internados, inclusive los ancianos, en un hospital general. El objetivo del estudio fue analizar el proceso de interacción del equipo de enfermería que cuida de los ancianos internados en hospitales de la $17^{\mathrm{a}}$ Coordinación Regional de Salud/Secretaria Estatal de Salud del Rio Grande do Sul. Es una investigación cualitativa e interpretativa cuyos datos fueron obtenidos por medio de entrevistas a 74 integrantes del equipo de enfermería: enfermeros, técnicos y auxiliares de enfermería de 14 hospitales. El análisis se llevó a cabo por medio de la interacción simbólica, resultando en categorías de significado de las interacciones y actitudes sobre: los profesionales de la enfermería, la vigencia en la falta de capacitación, el proceso del cuidado de los pacientes ancianos hospitalizados a partir de la forma en que el fenómeno se presenta en su vida social, y con respecto a la actitud, se considera la importancia de la capacitación especializada en gerontogeriatría, sin dejar de lado, la vocación personal para el cuidado de los ancianos como algo imprescindible.
\end{abstract}

DESCRIPTORES: Enfermería geriátrica. Anciano. Cuidados de la enfermería. Hospitalización. 


\section{INTRODUÇÃO}

O impacto do envelhecimento humano em toda a sociedade é visível, particularmente no sistema de saúde, no qual se constata déficit em sua infra-estrutura para atender as demandas desse estrato populacional, em termos de espaço físico, políticas, ações e intervenções específicas e, também, de recursos humanos capacitados qualitativa e quantitativamente.

Uma vez constituindo a equipe multiprofissional e interdisciplinar na área da saúde e, tendo em vista a atenção prestada às pessoas hospitalizadas, a enfermagem formada por enfermeiro, técnico e auxiliar é responsável pelo cuidado de todos os pacientes, incluindo os idosos que se encontram internados em hospitais gerais. Para isso, é necessário que esses profissionais tenham conhecimentos e habilidades próprias sobre como cuidar de idosos, além de ter afinidade e desejar trabalhar com a população dessa faixa etária, vislumbrando o desenvolvimento de uma prática de enfermagem qualificada e resolutiva.

Ao entender que o cuidar de pessoas idosas envolve conhecimentos, sentimentos, comportamentos e atitudes da enfermagem ao interagir com o ser receptor de cuidado, no caso a pessoa idosa, se considera que os preceitos do interacionismo simbólico podem ser utilizados para fundamentar a discussão desse estudo. Tal suporte teórico contempla o entendimento da complexa relação entre a sociedade e o indivíduo, o desenvolvimento de símbolos significantes e o processo de comportamento da mente a partir de uma orientação filosófica e de fatos da vida cotidiana. Nas interações sociais os indivíduos prevêem, em seu imaginário, condutas antecipadas de outrem e, freqüentemente, agem movidos por essas condutas. ${ }^{1-3}$

O significado dado por indivíduos sobre um fenômeno se dá mediante a interação do eu, da mente e da sociedade. Essa interação resulta da vida social, em cujo processo o indivíduo é um sujeito, um ator ativo. ${ }^{1-3}$ Assim, pode-se dizer que os profissionais de enfermagem processam o cuidado de idosos internados em hospitais gerais, com base na forma como o fenômeno se apresenta em sua vida social, em ambiente profissional e de como o interpretam. O cuidar constitui-se em um processo dinâmico que depende da interação e de ações delineadas a partir do conhecimento da realidade do idoso e sua família. ${ }^{4}$ Entendeu-se que tal processo possibilita olhar para a pessoa idosa, considerando os aspectos biopsicossociais e espirituais vivenciados pelo idoso e pela família.
Assim, este estudo teve por objetivo analisar o processo interacional dos integrantes da equipe de enfermagem ao cuidar de pessoas idosas que se encontram internadas em hospitais gerais, localizados na região de abrangência da $17^{\mathrm{a}}$ Coordenadoria Regional de Saúde/Secretaria Estadual de Saúde do Rio Grande do Sul (CRS/SES/RS).

\section{METODOLOGIA}

Em face da temática e de seu contexto, esse estudo constitui-se em pesquisa qualitativa com direcionamento de paradigma interpretativo.

O interacionismo simbólico ${ }^{1-3}$ foi a opção de suporte teórico-metodológico que permitiu compreender o fenômeno, observando-se especialmente suas premissas básicas: a) os seres humanos agem em relação as coisas, tomando por base o significado que as coisas tem para ele; b) o significado de tais coisas, às vezes surge de uma interação social que a pessoa tem com os seus iguais; c) esses significados são manipulados e modificados através de um processo interpretativo, usado pela pessoa para lidar com as coisas que ela encontra.

\section{Local do estudo, população e amostra}

Os participantes do estudo representam a equipe de enfermagem formada por enfermeiros, técnicos e auxiliares de enfermagem que atuam em 14 hospitais de abrangência da $17^{\mathrm{a}} \mathrm{CRS} / \mathrm{SES} / \mathrm{RS}$, dos quais sete classificados pelo Ministério da Saúdecomo locais, seis microrregionais e um regional. ${ }^{3}$ A área dessa coordenadoria abrange 20 municípios com um total de 224.168 habitantes $^{5}$ sendo $11,5 \%$ com 60 anos ou mais de idade, com índice de três pontos percentuais a mais em relação ao do país.

A amostra selecionada foi por conveniência, incluindo em sua composição pelo menos um enfermeiro de cada hospital. Nos hospitais microrregionais com mais de um enfermeiro, foi escolhido um deles; e na instituição hospitalar classificada como regional, foram incluídos enfermeiros responsáveis pelas duas unidades de internação clínica. A adoção deste último critério deu-se em função dessas unidades concentrarem maior número de internação de idosos. Já técnicos e auxiliares de enfermagem foram selecionados considerando a representatividade de ambas as categorias e atuação em diferentes turnos de trabalho. A seleção dos participantes ocorreu conforme sua presença no local, no momento da visita da pesquisadora nos hospitais. 
Foram 74 participantes: 15 enfermeiros, 39 técnicos e 20 auxiliares, com os seguintes critérios de inclusão: estar em pleno exercício de suas atividades; pertencer a uma das categorias da equipe de enfermagem e aceitar participar do estudo. Convém salientar que foram incluídos todos os hospitais e todas as categorias de enfermagem dos diferentes turnos de trabalho (matutino, vespertino e noturno). Para garantia do anonimato os entrevistados foram codificados com a letra E, seguida do número da entrevista.

\section{Obtenção e análise dos dados}

Os dados foram coletados por meio de entrevista aberta, no período entre setembro de 2005 e março de 2006, centrando o foco nas seguintes perguntas norteadoras: como você interage com os pacientes idosos da unidade de internação na qual você atua? Fale como você cuida desses pacientes idosos. Como é para você cuidar de pessoa idosa?

Devidamente anotadas e gravadas com a anuência dos participantes, as entrevistas foram realizadas no próprio hospital e durante o expediente, pois os funcionários que aceitaram participar foram autorizados, sem prejuízo do trabalho, interromper momentaneamente suas atividades laborais.

Para análise dos dados foi organizado o conjunto dos depoimentos, procurando reunir e destacar as tendências e padrões relevantes presentes no seu conteúdo. Assim, a análise temática desdobrou-se em três etapas principais: pré-análise, na qual foi organizado o conjunto de dados obtidos; descrição analítica, iniciada já na etapa de pré-análise, na qual o material foi estudado e orientado à luz dos preceitos do referencial teórico adotado; interpretação, que tomou como subsídio as informações já utilizadas desde a pré-análise e o contexto mais amplo da enfermagem enquanto prática interacional com os pacientes.

\section{Cuidados éticos de pesquisa com seres hu- manos}

O projeto da pesquisa foi aprovado pelo Comitê de Ética em Pesquisa da Pontifícia Universidade Católica do Rio Grande do Sul, parecer No $605 / 05$, e os entrevistados assinaram o Termo de Consentimento Livre e Esclarecido, conforme prevê a Resolução No 196/96 do Conselho Nacional de Saúde, que trata da pesquisa com seres humanos. Previamente a essa assinatura, a pesquisadora prestou todas as informações esclarecedoras sobre o estudo aos participantes.

\section{RESULTADOS E DISCUSSÃO}

Uma vez analisados os depoimentos, estes foram agrupados por convergência de conteúdo em duas categorias analíticas. A primeira referese à construção de significados que emergiram na interação da enfermagem ao prestar cuidados ao paciente idoso no período de internação; e a segunda versa sobre a necessidade de conhecimentos específicos, mas também de atributo pessoal de vocação para cuidar de idosos.

O cuidado dirigido à pessoa idosa constituise em uma área especializada da enfermagem, cuja prática exige qualidades como: capacidade para estabelecer relação terapêutica, habilidades para reconhecer os atributos e características pessoais do idoso, conhecimento das alterações físicas e psicossociais decorrentes do envelhecimento, competência clínica e técnica para o cuidado do idoso doente, capacidade de comunicação e disposição para trabalhar com pessoas idosas, entre outras. ${ }^{6}$

\section{O significado da interação da enfermagem no cuidado do paciente idoso hospitalizado}

As interações para as ações de cuidado podem resultar em gratificações mútuas, ao sentir-se gratificado pela ação desenvolvida, o profissional volta a cuidar na busca de novo reconhecimento, reforçando assim seu modo de agir. Essa situação atua como um feedback em que o paciente recebe o cuidado e gratifica a ação do profissional.

Quando estou cuidando deles, me vejo no lugar deles, você sentir assimé diferente, a gente vê a parte mais sensivel, [...] atende o lado psicológico e isso faz bem tanto para eles como para mim, isso é gratificante (E72).

Esse depoimento leva a admitir que há necessidade do profissional de enfermagem ter qualidades pessoais de vocação ou disposição para o cuidado de idosos, o que poderá proporcionar satisfação para ambos os envolvidos. Contudo não é o suficiente para o real atendimento integral dos pacientes, carecendo de conhecimentos e habilidades especializadas gerontogeriátricas.

Do ponto de vista do interacionismo simbólico, ao interagir o indivíduo se torna um objeto social para os outros e vice-versa, utilizando símbolos e direcionando o self, ambos se engajam em ação mental tomando decisões para modificar direções, compartilhar perspectivas, definir a realidade e a situação e assumir o papel do outro. ${ }^{3}$

A Enfermagem é uma profissão que, no campo da saúde, deveria estar apta a desenvolver 
ações efetivas e de impacto na atenção à vida e saúde das pessoas. $\mathrm{O}$ assistir envolve o profissional em suas interações pessoais e ambientais, identificando dificuldades no atendimento das necessidades básicas e estabelecendo intervenções com vistas a minimizar ou solucionar tais questões. ${ }^{4,6-7}$

Os participantes deste estudo interagiam à semelhança do que o interacionismo simbólico atribui, o de se colocar no lugar de outro, ou verse na posição do paciente e, consequentemente, imaginar como gostaria de ser cuidado, emergindo nesse relacionamento a empatia, como se pode perceber nesta afirmação: eu cuido sempre procurando passar o máximo [...] me ponho no lugar, como eu gostaria de ser tratada [...] dando toda a atenção. De repente até demais, [...] tendo bastante paciência (E41).

O hospital é, para os pacientes idosos, local estranho e ameaçador quando se submetem a diversos procedimentos diagnósticos e terapêuticos. Além disso, no período de internação, muitas vezes é necessário que permaneçam maior tempo em repouso e, em alguns casos, apresentam limitações de movimentos, levando à dependência e perda da autonomia. Em tal cenário, cabe à enfermagem estar atenta e procurar amenizar essa situação, demonstrando segurança, respeito e compreensão e, ao mesmo tempo incentivando a recuperação da autonomia pela prática possível do auto-cuidado.?

Neste estudo, a enfermagem percebia que os pacientes idosos requeriam mais tempo para os cuidados, porque gostavam de conversar, exigindo mais tempo junto deles para receber carinho e atenção, necessidades que os profissionais se dispunham a satisfazer e, além disso, manter uma relação que ultrapassava os cuidados costumeiros, numa conduta semelhante a que tem com seus parentes idosos.

Tenho muito carinho pelos idosos, [...] dou tempo para poder ficar junto deles. Eles gostam de puxar conversa, tentam te segurar [...]. É como se fossem meus pais (E3).

O que mais gosto neles é que eles querem transmitir uma atenção, assim como eles são carentes de atenção. Eles transmitem carinho para a gente, deixam transparecer isto. São carentes de carinho e gostam de passar isso também (E5).

Esses depoimentos e outros, sobre idosos carentes de atenção por falta de interação social, mostram como esses requerem cuidados mais diretos e demorados por parte de quem os cuida, mas eles reconhecem e retribuem com afetos a atenção que recebem.
A falta de capacitação especifica em enfermagem gerontogeriátrica, reconhecida pela enfermeira, mais uma vez, leva-a a condutas pouco recomendáveis. Seria o caso aqui de empreendimentos junto à família e comunidade, para a promoção da reinserção social por meio de incentivo de visitas de amigos, membros de igreja e voluntários, participação em grupos de convivência e outros programas de inclusão social.

Embora os atributos de respeito, valorização e afetividade pareciam estar presentes no cotidiano da equipe ao desenvolver as ações cuidativas, as particularidades concernentes ao cuidado específico, nos aspectos biológicos, psicossociais e culturais não estavam contempladas em suas manifestações, que pode ser atestada a seguir.

Desde que estou trabalhando na enfermagem eu sou assim: se eu vou cuidar, vou cuidar todos iguais, não tem discriminação de ser criança, meia-idade ou idoso. Eu sempre cuido do mesmo jeito e penso que todos têm que ser cuidados. Eu sempre tenho essa idéia de que eles estão aqui por alguma razão, eles não estão aqui por nada (E19).

Um dos fatores que contribuem para a boa interação entre profissionais de enfermagem e o idoso hospitalizado é a possibilidade do diálogo, que serve de mote para a formação de vínculo na prestação de cuidados.

Na perspectiva interacionista, essa interatividade em enfermagem usando a linguagem como instrumento, facilita ordenar a experiência para discriminar, generalizar e distinguir o ambiente. Desse modo, o mundo é, literalmente, caracterizado por significados atribuídos pelos indivíduos por meio da linguagem que modela o comportamento. ${ }^{3,8}$ Assim vejamos:

Gosto de atender pacientes idosos [...]. Na enfermagem, gosto mais de cuidar de idosos, porque a gente conversa (E6).

Embora seja um dos instrumentos básicos da Enfermagem no desenvolvimento de relacionamento terapêutico, ${ }^{9-10}$ a comunicação deve ser praticada pelo profissional com competência interpessoal para tal, deve levando em conta as especificidades de cada área de atuação.

Os sujeitos desta pesquisa realizavam a auto-reflexão, identificando-se e estabelecendo uma relação que procurava direcionar suas ações e executá-las da melhor forma, utilizando símbolos significantes, como por exemplo, usar uma brincadeira, para favorecer a aproximação e a 
formação de vínculo, dentro de suas experiências pessoais, seus conhecimentos profissionais mesmo com limitações.

Cuido dos pacientes idosos tentando fazer o que é possível para que se sintam bem, tenham confiança no atendimento. Muitas vezes, eles questionam o que na medida do possível eu respondo, mas a gente não sabe tudo (E21).

$\mathrm{Na}$ visão do paciente, os atributos que ele considera mais importantes estão voltados para os aspectos interpessoais no relacionamento com o profissional que lhe presta o cuidado. Esses aspectos se baseiam em receber atenção e amizade da equipe, que seja boa ouvinte e carinhosa, tenha competência e habilidade e que atenda rapidamente às chamadas. ${ }^{9}$

Assim, o cuidado de enfermagem é permeado de significações e, quando se trata de paciente idoso, parece haver um olhar especial para suas necessidades peculiares e sua vulnerabilidade, o que o torna merecedor de atenção diferenciada.

Tento cuidar do idoso da melhor maneira possível, com carinho, atenção e conversas, que é o principal. Vendo-o como um ser por inteiro e não uma peça adoentada ali em cima da cama (E5).

Quando o indivíduo é hospitalizado passa a participar de um grupo social específico de pessoas internadas, sendo-lhe impostos papéis caracterizados por acentuada dependência da equipe de saúde; espaço físico limitado ao seu quarto ou leito; abandono do uso de suas roupas e objetos pessoais, além de rígido horário para suas atividades da vida diária, entre outros. ${ }^{11-12}$ Nesse sentido, no processo de cuidado a pacientes idosos emergem algumas dificuldades, como a adaptação dos internos ao novo ambiente, de afastamento da família e capacidade para entender as inúmeras terminologias e exigências para as quais nem sempre foi preparado.

Para reconhecer o modo de ser do paciente idoso, a enfermagem, freqüentemente, age na tentativa de atender suas expectativas, proporcionando-lhe atenção, carinho, espaço de escuta e colocando-se no lugar dele. A interação profissional-paciente deve transcorrer em um espaço de respeito, em que atitudes e gestos promovam a dignidade, a auto-estima, a privacidade e a individualidade. Dentre as ações que reverenciam a dignidade e a autonomia do idoso, "[...] está a comunicação com o direito à informação". 13:741 $\mathrm{Na}$ fala da equipe deste estudo, identifica-se que a comunicação está presente ao mencionar que mantêm diálogo e fornece orientações, mesmo que limitadas, por falta de conhecimento já reconhecida por ela própria.

Gosto de dar orientações, como para minha vozinha, que não está doente, mas é um controle [...]. Penso que os que estão aqui no hospital também precisam... (E22).

A avaliação do cuidado prestado ao paciente baseia-se na qualidade dos cuidados instrumentais, os procedimentos técnico-científicos, e dos expressivos como o respeito, a confiança, a empatia e a comunicação aberta para a solução de problemas por parte do profissional no momento de prestar os cuidados. ${ }^{10}$ Enquanto cuidado instrumental, a presença do familiar acompanhante no ambiente hospitalar para o idoso internado, prevista na Portaria Ministerial, minimiza o desconforto no idoso do distanciamento de sua família, ao mesmo tempo em que oportuniza a aprendizagem do auto-cuidado após a alta, de ambos, o idoso e a família. Além de o familiar desenvolver atividades de cuidado do idoso em conjunto com a equipe no hospital, tornase um cliente e um parceiro da enfermagem. ${ }^{11-12}$

Contudo, a avaliação do cuidado expressivo da parte dos pacientes pode tomar contornos diversos, dependendo da visão e circunstâncias por que passa a pessoa. Para alguns, "ser bem cuidado" representava apenas receber atenção, carinho, amizade. No discurso dos profissionais de enfermagem também emergiram, principalmente, indicações de cuidado expressivo mais delimitado a dar atenção, carinho, paciência e dedicar mais tempo ao cuidado.

Os conhecimentos da enfermagem gerontogeriátrica, levariam a equipe a identificar diversos pontos que contribuiriam para que o paciente idoso recebesse a atenção necessária e, favorecesse um período de hospitalização com ações mais resolutivas de seus problemas de saúde. O diálogo, saber ouvir, estabelecer ambiente seguro, valorizar as queixas, fornecer orientações e apoio aos familiares e manter uma interação aberta e terapêutica, constituiriam condições favoráveis de tratamento e recuperação do paciente idoso hospitalizado. ${ }^{6-7,10}$ Tais especificidades de cuidado, contudo, não foram observadas nos depoimentos dos integrantes da equipe de enfermagem, denotando que suas ações profissionais junto aos idosos hospitalizados se davam apenas na base de relações sociais que se estabeleciam entre eles, como pares, mesmo porque apreciavam conviver com idosos mais do que com pessoas de outras idades. 
Conhecimento sobre o envelhecimento e interação com idosos no espaço hospitalar

É essencial que a equipe de enfermagem seja capacitada em gerontogeriatria para melhor desempenhar suas tarefas com o paciente idoso adquirir conhecimentos e desenvolver destrezas e habilidades específicas de cuidados, contemplando as características biológicas, psicossociais, culturais e espirituais de pessoas idosas. Do mesmo modo, é necessário conhecer políticas e programas voltados à população idosa e, responsabilizar-se pela qualidade da assistência/cuidado de enfermagem em seus diferentes níveis de atenção à saúde, com ações de promoção, proteção e reabilitação da saúde, na perspectiva da integralidade da assistência. ${ }^{13-14}$

Ao analisar as manifestações da enfermagem neste estudo, parece que a equipe reconhece a necessidade de ter conhecimentos atualizados, apontando a área da geriatria como exemplo para justificar tal manifestação, embora a maioria manifestasse não haver sido capacitada ou não ter ainda buscado especialização ou atualização.

Em relação ao desconhecimento de como proceder diante de situação envolvendo pessoa idosa, o ser humano, independente de sua formação e de sua posição, em situações desconhecidas usa mecanismos mentais e lança mão de experiências vividas, marcadas por símbolos, para apresentar soluções aos problemas que se apresentam. ${ }^{15}$ Desse modo agiam os integrantes da equipe de enfermagem deste estudo, que referiam não terem conhecimento suficiente para a sua plena atuação profissional.

Então, deveríamos estudar para poder ajudar [...] não que a gente não aprenda, um diz uma coisa, outro diz outra, mas o certo seria a gente estudar (E59).

A geriatria e a gerontologia compõem uma área emergente, na qual ainda há um reduzido número de profissionais da saúde com formação, qualificação ou capacitação. Acredita-se que no conjunto das ações previstas para a formação e atualização desses profissionais, via educação permanente, estejam sendo incluídos conhecimentos gerontogeriátricos. Esse entendimento surge não somente no imaginário dos responsáveis pela formação de recursos humanos, mas naqueles profissionais que cotidianamente exercem sua prática assistencial.

Acho que precisa ter conhecimento [...], tem bastante idosos internados e a gente não estuda muito sobre isso, estuda-se sobre criança, mulher, adulto, mas sobre idoso não. E eles querem atenção, aqui é um hospital pequeno e a gente vê isso, quem reclama nem é o próprio paciente, mas o familiar (E61).

Quanto à estruturação dos serviços hospitalares para atendimento de idosos, há certo consenso sobre os princípios e características que devam permeá-los, com importantes implicações sobre os recursos e os tipos de profissionais necessários, intrinsecamente relacionados à natureza das questões de saúde das pessoas idosas. Enfatiza-se ainda que, dentre as características do processo saúde-doença, quando se trata de pessoa idosa, estão presentes - múltiplos problemas de saúde; polifarmácia; rápida deterioração em caso de morbidades agudas quando não adequadamente tratadas; presença de doenças inespecíficas e insidiosas; complicações secundárias a doenças e tratamentos; fatores sociais e ambientais frequentemente envolvidos no desenvolvimento, na descompensação e na recuperação de problemas de saúde. ${ }^{16}$

Tal quadro indica a importância dos serviços e dos recursos humanos especializados em gerontogeriatria, com abordagem interdisciplinar, e nos casos agudos em que há necessidade de hospitalização do idoso, o serviço deve proporcionar também um atendimento multiprofissional. Desse modo, profissionais com conhecimento e familiarizados com as particularidades de manejo das doenças em idosos podem melhor incorporar habilidades e intervenções que não as estritamente técnicas gerais. Além disso, têm condições de identificar idosos com maior risco de complicações e de evolução desfavorável, oferecendo serviço de reabilitação com vistas à rápida recuperação de sua saúde, abreviando o período de internamento, e a adequada preparação do idoso e sua família para a alta hospitalar, garantindo a continuidade da assistência no âmbito da rede básica de saúde. ${ }^{16-17}$

Alguns membros da equipe reconhecem que, além dos conhecimentos específicos técnicocientíficos, há a necessidade de adotar conduta profissional adequada, de natureza expressiva, para atuar com pessoas idosas. Mencionaram que tal segmento requer atenção especial, apontando ainda como demanda os aspectos relativos à compreensão do processo de morte e luto. Consideram que essa é uma condição que mais se aproxima das pessoas idosas, razão pela qual deveriam estar adequadamente preparados para atendê-la.

Cuidar de idoso, eles que são pessoas mais frágeis, mais doentes, pessoas muito carentes de tudo. [...] e ainda com problema psicológico [...] é, [...] a gente deveria fazer cursos nesta área (E28). 
Penso que precisam de um cuidado diferenciado, [...] de atenção psicológica, até para a preparação para a morte, [...]. É preciso entender e atender o paciente idoso; é diferente de outros, a gente não sabe como agir (E42).

Portanto, pode-se dizer que ter conhecimento acerca do processo de envelhecimento, políticas de atenção ao idoso, prevenção de incapacidades funcionais, promoção de um envelhecimento saudável, tratamento e reabilitação de morbidades já instaladas são elementos hoje indispensáveis aos profissionais de enfermagem responsáveis pelo cuidado de idosos, independente de qualquer tipo serviço de saúde. E também para que possam direcionar seu pensamento para fazer da enfermagem gerontogeriátrica um novo modo de exercer sua práxis. ${ }^{4,6-7}$

A doença e a hospitalização, quase sempre apresentam uma experiência desafiadora que necessita de ajustamento a mudanças e perdas. Mudança de ambiente, de vida e de hábitos; perda da saúde, do controle sobre si mesmo e sobre o ambiente. A doença e a hospitalização provocam, ainda, tanto no paciente quanto nos familiares - medo do desconhecido; questionamentos a respeito da vida e da morte, a respeito de valores e sentido da vida; dúvidas quanto a continuar vivendo com qualidade ou a dura realidade de enfrentar limitações inesperadas. ${ }^{10}$

Em tal cenário, a equipe de enfermagem não deveria eximir-se de prestar atendimento às necessidades psicoespirituais, em especial, nas situações de cronicidade, quando existe sofrimento e dor por longo período. Convém lembrar que para ajudar o idoso a recuperar a independência e autonomia e alcançar uma velhice ativa e saudável, é necessário que os profissionais transcendam a abordagem clínico-curativa, atuando com postura interprofissional e interdisciplinar. Para isso, é preciso implantar e consolidar práticas que contemplem as particularidades da atenção à saúde e à vida das pessoas idosas, com base nos princípios fundamentais de universalidade, equidade e integralidade requeridos no Sistema Único de Saúde. ${ }^{13-14}$

Desse modo, a equipe de enfermagem buscaria atender as especificidades de cuidado do idoso, integrando o cuidado instrumental e o expressivo na medida adequada às reais necessidades de atenção. Contudo, a velhice pode tomar um caráter conflitivo, não só para o próprio idoso, mas também para as pessoas que o cuidam. O grau de conflito representado para cada pessoa e as condutas defensivas que adotam para evitá-lo, guarda relação com as experiências pessoais prévias. Os cuidados de idosos enfermos com incapacidades prolongadas podem conduzir inconscientemente, integrantes da equipe de enfermagem a se defrontar com seus próprios temores do envelhecimento com o sofrimento e com a morte. Nessa situação, é importante que os profissionais se submetam ao exercício de autoconhecimento para trabalhar seu processo interno quanto a preconceitos, crenças e dificuldades em relação à velhice, doença e sofrimento a favor de uma saúde mental positiva e, consequentemente, tornar-se um adequado cuidador profissional. ${ }^{17}$

Segundo os preceitos do interacionismo simbólico, os indivíduos se interagem e a sociedade, por extensão, é constituída de indivíduos interagindo entre si. Assim, o ser humano é entendido como um ser agindo no presente, influenciado pelo que houve no passado e pelo que está ocorrendo no presente. Cabe lembrar que interação não se reduz somente ao que está acontecendo entre as pessoas, mas também abrange o que acontece no seu interior. ${ }^{3}$

As manifestações dos participantes desta pesquisa dão a entender que, para desempenhar ações do cuidado a idosos, o profissional deve ter, além de conhecimentos específicos, atributos como vocação, predisposição, vontade e gostar de trabalhar com indivíduos que se encontram na velhice. Por exemplo: quando o profissional gosta de trabalhar com idosos, ele cuida, gosta daquilo que faz e tem vontade de cuidar, essa é a verdade (E57).

Ao responsabilizar-se pelos cuidados no contexto hospitalar, a enfermagem deve ser capaz de garantir atenção individualizada aos idosos e atender suas necessidades, considerando as limitações e recursos para recuperação de sua autonomia e independência, além de buscar interagir com os profissionais de outras áreas para abranger todas as dimensões de atenção ao idoso enfermo. Assim, na área gerontogeriátrica o profissional deve ter competência especifica, ter vocação e ser humilde para aprender também com o próprio idoso, principalmente se o profissional é jovem.

\section{CONSIDERAÇÕES FINAIS}

$\mathrm{Na}$ análise do processo interacional dos membros da equipe de enfermagem, ao cuidar de pessoas idosas hospitalizadas no contexto da região de abrangência da $17^{\mathrm{a}} \mathrm{CRS} / \mathrm{SES} / \mathrm{RS}$, foi possível identificar significados atribuídos que demonstram falta de preparo profissional especializado. 
Quanto à atitude, consideraram a importância de o profissional possuir conhecimentos especializados em gerontogeriatria, embora reconhecessem sua não capacitação ou especialização e as limitações de suas ações profissionais; mas enfatizaram a importância da vocação pessoal para o cuidar de pessoas idosas.

Assim, pode-se concluir que os profissionais de enfermagem processam o cuidado dos pacientes idosos hospitalizados a partir da forma como o fenômeno se apresenta em sua vida social. Dada à falta de capacitação da equipe em gerontogeriatria, não houve a relação profissional especializada na construção de interações significantes de cuidados da vida e saúde de pacientes idosos.

As interpretações dadas e nominadas em alguns significados e atitudes, tiveram base nas interações sociais estabelecidas entre os profissionais da enfermagem e os pacientes idosos como se fossem pares numa comunidade, embora ambos detivessem seus papéis próprios, o de paciente de um lado, e o da enfermagem do outro.

Tais significados e atitudes identificados revelam a necessidade premente de capacitação do corpo de enfermagem hospitalar para o cuidado de pacientes idosos, como também fornecem subsídios orientadores no aprofundamento de estudos de qualidade de serviços de saúde, principalmente dirigidos aos resultados de interações profissionais que visam às mudanças de comportamento de saúde e de estilo de vida das pessoas idosas.

\section{REFERÊNCIAS}

1. Haguette TMF. Metodologias qualitativas na sociologia. $4^{\mathrm{a}}$ ed. Petrópolis (RJ): Vozes; 1995.

2. Blumer H. Symbolic Interacionism: perspective and method. California (US): Prentice-Hall, 1969.

3. Charon JM. Symbolic interacionism: An introduction, an interpretation, an integration. Eighth edition. Upper Sadle River, New Jersey (US): Pearson Prentice Hall, 2004.

4. Duarte YAO. Princípios de assistência de enfermagem gerontológica. In: Papaléo Netto M. Gerontologia: a velhice e o envelhecimento em visão globalizada. Rio de Janeiro (RJ): Atheneu, 1996.

5. Brasil. Anuário Estatístico de Saúde do Brasil. Fundação Sistema Estadual de Análise de Dados.
Disponível em: portal.saude.gov.br/portal/ anuario2001, 2001.

6. Eliopoulos C. Enfermagem gerontológica. $5^{\mathrm{a}}$ ed. São Paulo (SP): Artmed, 2005.

7. Gonçalves LHT, Alvarez AM. O Cuidado na enfermagem gerontogeriátrica: Conceito e prática. In. Freitas EV, Py L, Cançado FAX, Doll J, Gorzoni ML. Tratado de Geriatria e Gerontologia, $2^{\mathrm{a}}$ ed. Rio de Janeiro (RJ): Guanabara Koogan, 2006. p. 1110-6.

8. Lopes CHAF, Jorge MSB. Interacionismo simbólico e a possibilidade para o cuidar interativo em enfermagem. Rev Esc Enferm USP. 2005 Jun. 39(1):103-8.

9. Stefanelli MC. Ensino de técnicas de comunicação terapêutica enfermeira-paciente: referencial teórico (parte II). Rev Esc Enferm USP. 1987 Ago; 21(2):107-15.

10. Oliveira AML, Girardello EB. Satisfação do paciente com os cuidados de enfermagem: comparação entre dois hospitais Rev Esc Enferm USP. 2006 Mar.; 40(1):71-7.

11. Leite MT. Cuidando do idoso hospitalizado: a experiência do familiar (Série dissertações de mestrado). Ijuí (RS), Ed. Unijui; 2000.

12. Schier J, Gonçalves LHT, Lima MGO. Programa de acompanhante hospitalar para paciente geriátrico. Rev Gaúcha Enferm. 2003 Abr; 24(1):61-8.

13. Brasil. Ministério da Saúde Portaria No 2.528 de 19 de outubro de 2006. Política Nacional de Saúde da Pessoa Idosa. Disponível em: http:// portal.saude. gov.br/portal/arquivos/pdf/

14. Brasil. Ministério da Saúde. Secretaria Executiva. Subsecretaria de Planejamento e Orçamento. Plano Nacional de Saúde: um pacto pela saúde no Brasil. Brasília: 2005. Disponível em: http:/ / dtr2001.saude. gov.br/editora/produtos/livros/pdf/

15. Leonart E, Mendes MMR. Gerontological training of nursing technicians: a cultural approach. Rev LatinoAm. Enfermagem. 2005 Jul-Ago. 13(4):538-46.

16. Coelho Filho JM. Modelos de serviços hospitalares para casos agudos em idosos. Rev Saúde Pública. 2000 Dez; 34(6):666-71.

17. Lenardt MH, Hammerschmidt KSA, Pivaro ABR, Borghi ACS. Os idosos e os constrangimentos nos eventos da internação cirúrgica. Texto Contexto Enferm. 2007 Out-Dez. 6(4):737-45.

18. Gonçalves LHT, Scheier J. "Grupo aqui e agora" uma tecnologia leve de ação sócio-educativa de enfermagem. Texto Contexto Enferm. 2005 AbrJun. 14(2):271-9. 